wissen kompakt 2014 · 9:2-4 DOI 10.1007/s11838-014-0229-8

Online publiziert: 18. Dezember 2014

(c) Springer-Verlag und Freier Verband Deutscher Zahnärzte e.V. 2014

\title{
B. Haller
}

Klinik für Zahnerhaltungskunde und Parodontologie, Universitätsklinikum Ulm

\section{Zahnmedizinische Forschung in der Zwickmühle - am Beispiel der restaurativen Therapie}

Bei den rund 55 Mio. Füllungen, die jährlich in Deutschland gelegt werden, handelt es sich in etwa 70\% der Fälle nicht um Erstversorgungen, sondern um den Ersatz defekter, bestehender Füllungen. Wie sieht es hier mit der Eignung von Kompositen aus, die bei der minimalinvasiven Erstversorgung Mittel der Wahl sind? Wann sind indirekte Restaurationen mit Einbeziehung der Höcker vorzuziehen, und nach welchen Kriterien soll die Entscheidung getroffen werden? Bei diesen Fragen wird in der Regel auf den Bedarf bzw. den Mangel an kontrollierten klinischen Studien hingewiesen. Dahinter verbergen sich jedoch mehrere Fragen.

\section{》) Klinische Langzeitstudien hinken der Versorgungsrealität immer hinterher}

Zum einen steigt mit der Beobachtungsdauer einer klinischen Studie immer auch die Wahrscheinlichkeit, dass die verwendeten Materialien zum Zeitpunkt der Publikation gar nicht mehr aktuell und praxisüblich sind. Klinische Langzeitstudien hinken der Versorgungsrealität immer hinterher. Wenn zum Beispiel eine 2007 publizierte Studie nach einer Beobachtungsdauer von 15 Jahren zu dem Schluss kommt, dass Vollkronen bei der Versorgung komplexer, mehrflächiger Kavitäten eine höhere Überlebensrate aufweisen als direkte Kompositrestaurationen, dann reflektieren diese Ergebnisse in Bezug auf die verwendeten Restaurationstechniken (u. a. Instrumente, Materialien) den Stand von etwa 1987 [1].
Aufgrund dieses Dilemmas, das seit Langem bekannt ist, werden zahlreiche In-vitro-Studien durchgeführt, um das Potenzial von Füllungsmaterialien im Hinblick auf Abdichtungsvermögen, mechanische Festigkeit, Verschleiß, Stabilisierung der Restzahnsubstanz u. v. m. abzuschätzen. Unklar ist, wie sich die Resultate von In-vitro-Studien auf die klinische Realität übertragen lassen. Dabei ist aus den genannten Gründen offensichtlich, dass es ohne valide In-vitro-Studien nicht geht. Allein schon deshalb, weil so viele Variablen zu untersuchen sind, von der Kavitätenpräparation bis zur Schichttechnik, die alle zu einer Optimierung beitragen könnten. Die Konsequenz liegt auf der Hand: Es müssen neue, geeignete In-vitro-Modelle etabliert werden, die eine zuverlässige Abschätzung des klinischen Potenzials von Restaurationsverfahren möglich machen.

Die entscheidende Frage ist: Wer soll diese Forschung finanzieren? Öffentliche Fördereinrichtungen wie die Deutsche Forschungsgemeinschaft (DFG) legen schon seit geraumer Zeit ihren Schwerpunkt auf die sog. Grundlagenforschung, wo hauptsächlich molekularbiologische Forschungsansätze eine Rolle spielen. Deren klinische Relevanz erschließt sich oft weder auf den ersten noch auf den zweiten Blick. Die Zahnmedizin hat inzwischen gelernt, dass an öffentliche Fördertöpfe oft nur zu kommen ist, wenn man sich diesem Mainstream anschließt. Es ist zu bedauern und stimmt bedenklich, dass die Forschung zu ureigenen zahnmedizinischen Anliegen auf der Strecke zu bleiben droht. Dabei wäre es - um beim Thema zu bleiben - durchaus von volkswirtschaftli- 
chem Interesse, Mittel und Wege zu finden, um die Liegedauer von Restaurationen zu verlängern. Der Hinweis, man solle sich doch wegen einer Forschungsförderung an die Industrie wenden, ist nett gemeint, aber nicht wirklich hilfreich. Öffentliche Drittmittelgeber, Dentalindustrie und der zahnärztliche Berufsstand mit seinen Vertretungen sind hier gemeinsam gefordert.

\section{》) Die Forschung zu \\ zahnmedizinischen Anliegen droht auf der Strecke zu bleiben}

Es ist an der Zeit, dass die zahnmedizinische Forschung sich wieder stärker auf ihre eigenen Themen konzentriert und die Lösung zahnmedizinisch relevanter Probleme ins Visier nimmt. Die Ausrichtung an den eher zahnmedizinfernen Forschungsschwerpunkten der medizinischen Fakultäten und die Reduzierung auf mögliche Assoziationen zwischen oralen Veränderungen und allgemeiner Gesundheit wirkt gelegentlich opportunistisch und wird dem Forschungsbedarf zu zahnmedizinisch relevanten Themen nicht gerecht. Vor allem helfen sie dem Zahnarzt nicht bei seinen Therapieentscheidungen. Sollte sich dieser Trend fortsetzen, bedeutet dies langfristig nicht eine Stärkung der Zahnmedizin, sondern eine Schwächung. Die Frage nach der Haltbarkeit von Restaurationen und den Möglichkeiten, diese zu verbessern, steht dabei nur exemplarisch für einen Missstand, den es zu beseitigen gilt.

\section{3. haver}

Prof. Dr. Bernd Haller

\section{Korrespondenzadresse}

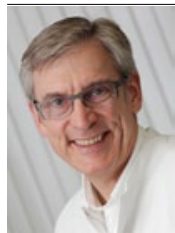

Prof. Dr. B. Haller

Klinik für

Zahnerhaltungskunde

und Parodontologie,

Universitätsklinikum Ulm

Albert-Einstein-Allee 11,

$89081 \mathrm{Ulm}$

b.haller@uniklinik-ulm.de
Interessenkonflikt. B. Haller gibt an, dass kein Interessenkonflikt besteht.

\section{Literatur}

1. Miyamoto T, Morgano SM, Kumagai T et al (2007) Treatment history of teeth in relation to the longevity of the teeth and their restorations: outcomes of teeth treated and maintained for 15 years. J Prosthet Dent 97(3):150-156

\section{Infektiologie und Prophylaxe}

Infektiologie, die Behandlung von Infektionen, erfordert, neben der Kenntnis der Mikrobiologie, immer auch den Blick des Klinikers. Keinesfalls kann die Indikation für eine Antibiotikatherapie oder die Entscheidung, welche Substanz verwendet wird, nur auf der Basis mikrobiologischer Befunde getroffen werden. Die Einschätzung der klinischen Relevanz und die Kenntnis des

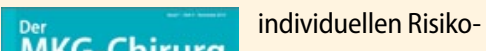

MKG-Chirurg individuellen Risikoprofils des Patienten müssen in der Hand des Arztes liegen. Die Ausgabe 4/2014 der Zeitschrift Der MKG-Chirurg widmet sich dem Schwer-

punkt "Infektiologie und Prophylaxe“ in folgenden Beiträgen:

- Infektiologie zwischen Klinik und Labor

- Odontogene Infektionen und Erregerspektren in der MKG-Chirurgie

- Perioperative Antibiotikaprophylaxe in der MKG-Chirurgie

- Lokale Antibiotika und Antiseptika

- Medizinhygieneverordnungen

Bestellen Sie diese Ausgabe zum Preis von 53,- EUR zzgl. Versandkosten bei Springer Customer Service Center Kundenservice Zeitschriften

Haberstr. 7

69126 Heidelberg

Tel.: +49 6221-345-4303

Fax: +49 6221-345-4229

E-Mail: leserservice@springer.com

Suchen Sie noch mehr zum Thema? Mit

e.Med, dem Online-Paket von Springer Medizin, können Sie schnell und komfortabel in über 500 medizinischen Fachzeitschriften recherchieren.

Weitere Infos unter springermedizin.de/ eMed. 


\section{Hier steht eine Anzeige.}

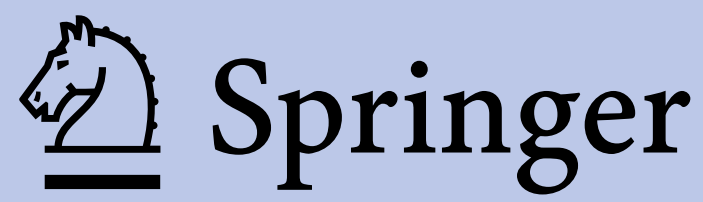

